\title{
Optical emission from GRB 050709: a short/hard GRB in a star-forming galaxy ${ }^{\star}$
}

\author{
S. Covino ${ }^{1}$, D. Malesani ${ }^{2}$, G. L. Israel ${ }^{3}$, P. D’'Avanzo ${ }^{1,4}$, L. A. Antonelli $^{3}$, G. Chincarini ${ }^{1,5}$, D. Fugazza ${ }^{1}$,
} M. L. Conciatore ${ }^{3}$, M. Della Valle ${ }^{6}$, F. Fiore ${ }^{3}$, D. Guetta ${ }^{3}$, K. Hurley ${ }^{7}$, D. Lazzati ${ }^{8}$, L. Stella ${ }^{3}$, G. Tagliaferri ${ }^{1}$, M. Vietri ${ }^{9}$, S. Campana ${ }^{1}$, D. N. Burrows ${ }^{10}$, V. D'Elia ${ }^{3}$, P. Filliatre ${ }^{11,12}$, N. Gehrels ${ }^{13}$, P. Goldoni ${ }^{11,12}$, A. Melandri ${ }^{3,14}$, S. Mereghetti ${ }^{15}$, I. F. Mirabel ${ }^{16}$, A. Moretti ${ }^{1}$, J. Nousek ${ }^{10}$, P. T. O’Brien ${ }^{17}$, L. J. Pellizza ${ }^{13}$, R. Perna ${ }^{8}$, S. Piranomonte ${ }^{3}$, P. Romano ${ }^{1}$, and F. M. Zerbi ${ }^{1}$

1 INAF, Osservatorio Astronomico di Brera, via E. Bianchi 46, 23807 Merate (LC), Italy e-mail: covino@mi.astro.it

2 International School for Advanced Studies (SISSA-ISAS), via Beirut 2-4, 34014 Trieste, Italy

3 INAF, Osservatorio Astronomico di Roma, via di Frascati 33, 00040 Monteporzio Catone (Roma), Italy

4 Dipartimento di Fisica e Matematica, Università dell'Insubria, via Valleggio 11, 22100 Como, Italy

5 Università degli studi di Milano-Bicocca, Dipartimento di Fisica, piazza delle Scienze 3, 20126 Milano, Italy

6 INAF, Osservatorio Astrofisico di Arcetri, largo E. Fermi 5, 50125 Firenze, Italy

7 University of California, Berkeley, Space Sciences Laboratory, Berkeley, CA 94720-7450, USA

8 JILA, University of Colorado, 440 UCB, Boulder CO 80309-0440, USA

9 Scuola Normale Superiore, piazza dei Cavalieri 7, 56126 Pisa, Italy

${ }_{10}$ Department of Astronomy \& Astrophysics, Pennsylvania State University, State College, PA 16801, USA

11 Laboratoire Astroparticule et Cosmologie, UMR 7164, 11 Place Marcelin Berthelot, 75231 Paris Cedex 05, France

12 Service d'Astrophysique, DSM/DAPNA, CEA Saclay, 91911 Gif-sur-Yvette Cedex, France

13 NASA, Goddard Space Flight Center, Greenbelt, MD 20771, USA

14 Università degli Studi di Cagliari, Dipartimento di Fisica, 09042 Monserrato (Ca), Italy

15 INAF/IASF Milano "G. Occhialini”, via E. Bassini 15, 20133 Milano, Italy

16 European Southern Observatory - Vitacura, Casilla 19001, Santiago 19, Chile

17 X-Ray \& Observational Astronomy Group, Dept. of Physics \& Astronomy, University of Leicester, Leicester LE1 7RH, UK

Received 7 September 2005 / Accepted 21 December 2005

\section{ABSTRACT}

We present optical observations of the short/hard gamma-ray burst GRB 050709, the first such event with an identified optical counterpart. The object is coincident with a weak X-ray source and is located inside a galaxy at redshift $z=0.1606 \pm 0.0002$. Multiband photometry allowed us to study the broad-band spectral energy distribution. Late-time monitoring places strong limits on any supernova simultaneous with the GRB. The host galaxy is not of early type. Spectra show that the dominant stellar population is relatively young ( 1 Gyr), and that ongoing star formation is present at a level of $2-3 L / L_{*} M_{\odot} \mathrm{yr}^{-1}$. This is at least 2 orders of magnitude larger than that observed in the elliptical hosts of the short GRB 050509B and GRB 050724. This shows that at least some short GRBs originate in a young population. Short/hard GRB models based on the merger of a binary degenerate system are compatible with the host galaxy characteristics, although there is still the possibility of a connection between young stars and at least a fraction of such events.

Key words. radiation mechanisms: non-thermal - gamma rays: bursts - gamma rays: individual GRB 050709

\section{Introduction}

Gamma-ray bursts (GRBs) are short pulses of gamma rays occurring at random positions in the sky. Two classes of GRBs are currently known, characterised by different durations and spectral properties (Kouveliotou et al. 1993). Long GRBs (typically lasting 10-100 s) are on the average softer than the short

* Based on observations carried out at ESO telescopes under programmes Id 075.D-0787 and 075.D-0468. ones (duration $<2 \mathrm{~s}$ ). Over the past years, great advances have been made in understanding the former class, thanks to the observation of their optical and radio counterparts. However, until recently, no optical emission from short GRBs had been identified (Hurley et al. 2002), leaving fundamental questions about their nature, progenitors and distances unanswered.

Recently, thanks to the Swift and HETE-2 satellites, accurate and rapid localisations of short GRBs have become available, enabling deep, sensitive searches at long wavelengths. 


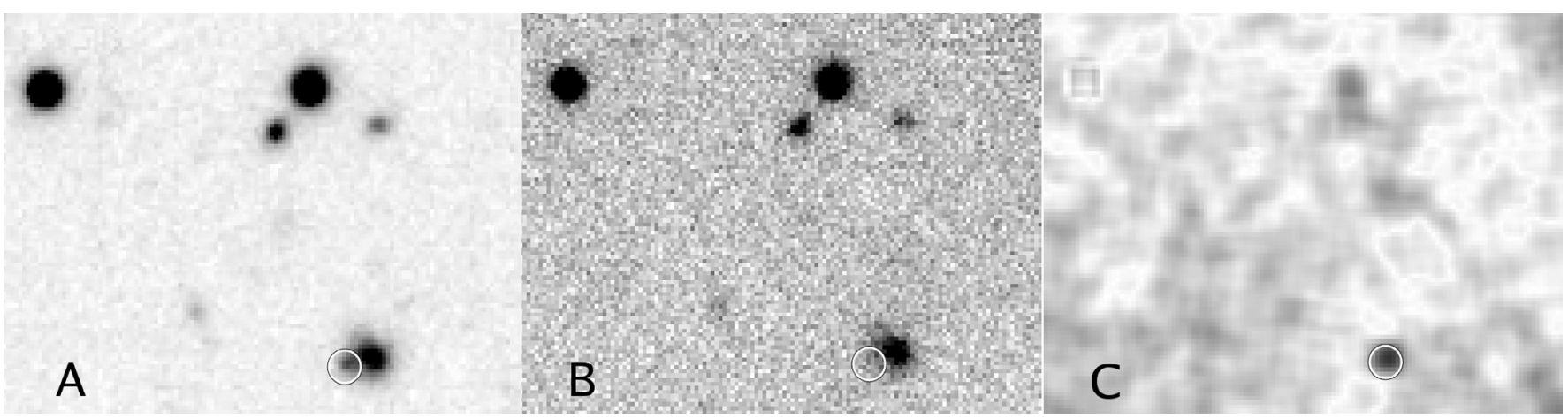

Fig. 1. $R$-band image of the field of GRB 050709, on 2005 Jul. 12.41 (A) and 20.42 (B). Panel C shows the result of the subtraction, evidencing a fading source coincident with the Chandra counterpart (circle). The boxes cover a region $30^{\prime \prime} \times 20^{\prime \prime}$ wide.

Swift discovered a weak X-ray counterpart to GRB 050509B (Gehrels et al. 2005), located $\sim 11^{\prime \prime}$ away from a bright elliptical galaxy at $z=0.2248$ (Bloom et al. 2005; Hjorth et al. 2005a; Castro-Tirado et al. 2005). An early-type galaxy (Gorosabel et al. 2005) is also associated with GRB 050724 (Barthelmy et al. 2005) at $z=0.257$ (Prochaska et al. 2005), the only other event for which an optical afterglow was singled out inside the host (Berger et al. 2005a; D'Avanzo et al. 2005). Finally, the line of sight of GRB 050813 (Retter et al. 2005) lies towards a galaxy cluster at $z=0.722$. A few elliptical galaxies were identified inside the XRT error circle, again supporting the association with early-type galaxies (Gladders et al. 2005; Berger 2005b; Prochaska et al. 2005).

GRB 050709 was discovered by HETE-2 on 2005 Jul. 9.94209 UT (Villasenor et al. 2005). Its prompt emission consisted of a single pulse lasting $70 \mathrm{~ms}$ in the 3-400 keV energy band, followed by a weaker, soft bump $\sim 100 \mathrm{~s}$ long. This second episode may be due to the afterglow onset (Villasenor et al. 2005), or to flaring activity (Barthelmy et al. 2005; King et al. 2005; Perna et al. 2005). In any case, the prompt emission properties are consistent with those of a short/hard GRB.

Follow-up observations with the Chandra X-ray observatory revealed a faint, uncatalogued X-ray source inside the HETE-2 error circle (Fox et al. 2005a). At the coordinates $\alpha_{\mathrm{J} 2000}=23^{\mathrm{h}} 01^{\mathrm{m}} 26^{\mathrm{s}} .9, \delta_{\mathrm{J} 2000}=-38^{\circ} 58^{\prime} 39^{\prime \prime} .5\left(0^{\prime} .4\right.$ error), it was coincident with a pointlike object embedded in a bright galaxy (Jensen et al. 2005) at $z=0.16$ (Price et al. 2005a). The variability of this source led Price et al. (2005b) to propose it as the optical counterpart of GRB 050709.

\section{Observations and data analysis}

We observed the field of GRB 050709 with the ESO Very Large Telescope (VLT), using the FORS 1 and FORS 2 instruments. In our first images, taken on 2005 Jul. 12, the pointlike object reported by Jensen et al. (2005) was clearly visible in the $V$ and $R$ bands (Fig. 1). Its coordinates are $\alpha_{\mathrm{J} 2000}=$ $23^{\mathrm{h}} 01^{\mathrm{m}} 26.96, \delta_{\mathrm{J} 2000}=-38^{\circ} 58^{\prime} 39^{\prime} \cdot 3\left(0^{\prime} \cdot 25\right.$ error), fully consistent with the Chandra position. The object lies inside a bright galaxy $(\approx 1,2$ away from its nucleus), whose magnitudes are $V=21.35 \pm 0.07, R=21.08 \pm 0.07$, and $I=20.63 \pm 0.08$. To search for brightness variations, we monitored the field at several epochs. Data analysis was performed by adopting a
Table 1. Observation log and photometry of the transient source. Errors are at the $1 \sigma$ confidence level, while upper limits are at $3 \sigma$. Data were not corrected for Galactic extinction.

\begin{tabular}{ccrcr}
\hline \hline $\begin{array}{c}\text { Date } \\
(\mathrm{UT})\end{array}$ & Instrument & $\begin{array}{r}\text { Exp. } \\
(\mathrm{min})\end{array}$ & Filter & Magnitude \\
\hline $2005 / 07 / 1209: 44$ & FORS 2 & 6 & $V$ & $24.38 \pm 0.10$ \\
$2005 / 07 / 1407: 21$ & FORS 1 & 6 & $V$ & $>25.00$ \\
$2005 / 07 / 2010: 16$ & FORS 1 & 6 & $V$ & (reference) \\
$2005 / 07 / 3002: 37$ & FORS 2 & 9 & $V$ & $>25.20$ \\
$2005 / 07 / 1209: 57$ & FORS 2 & 5 & $R$ & $23.83 \pm 0.07$ \\
$2005 / 07 / 2010: 07$ & FORS 1 & 6 & $R$ & (reference) \\
$2005 / 07 / 3002: 54$ & FORS 2 & 50 & $R$ & $>25.00$ \\
$2005 / 07 / 1209: 32$ & FORS 2 & 10 & $I$ & $>23.25$ \\
$2005 / 07 / 1407: 32$ & FORS 1 & 5 & $I$ & $>24.10$ \\
$2005 / 07 / 1806: 38$ & FORS 1 & 20 & $I$ & (reference) \\
$2005 / 07 / 3004: 10$ & FORS 2 & 9 & $I$ & $>23.50$ \\
\hline
\end{tabular}

subtraction technique (Alard \& Lupton 1998), well suited to identify variable objects even when blended with nearby sources. The pointlike object was found to be variable (at the $\sim 10 \sigma$ level), being undetectable from Jul. 14 onwards. This confirms the independent finding of Hjorth et al. (2005b). Magnitudes of the variable source were computed assuming a negligible flux in the reference epoch (see Table 1). Photometry of the transient was performed by inserting artificial stars of known brightness and calibrated by observing Landolt standard fields.

On Jul. 30 we took medium-resolution spectra of the host galaxy. Observations were carried out with the FORS 2 instrument at the VLT-UT1, with the $300 \mathrm{~V}$ grism, covering the wavelength range $6000-9200 \AA$ ( $6 \AA F W H M)$. From the detection of several emission lines, among them $\mathrm{H} \alpha, \mathrm{H} \beta$, and [O II], we derived a redshift $z=0.1606 \pm 0.0002$. This is consistent with the results of Fox et al. (2005b). Therefore, the rest-frame $B$-band luminosity of the host is ${ }^{1} L_{\mathrm{B}} \sim 3.5 \times 10^{42} \mathrm{erg} \mathrm{s}^{-1}\left(\sim 0.10 L_{*}\right.$, assuming $M_{B}^{*}=-20.13$ as determined from the SDSS survey; Blanton et al. 2003) and the candidate afterglow lies at a projected distance of $\approx 3.3 \mathrm{kpc}$ from the galaxy core.

\footnotetext{
1 Assuming a cosmology with $\Omega_{\mathrm{m}}=0.3, \Omega_{\Lambda}=0.7$, and $h_{0}=0.71$.
} 


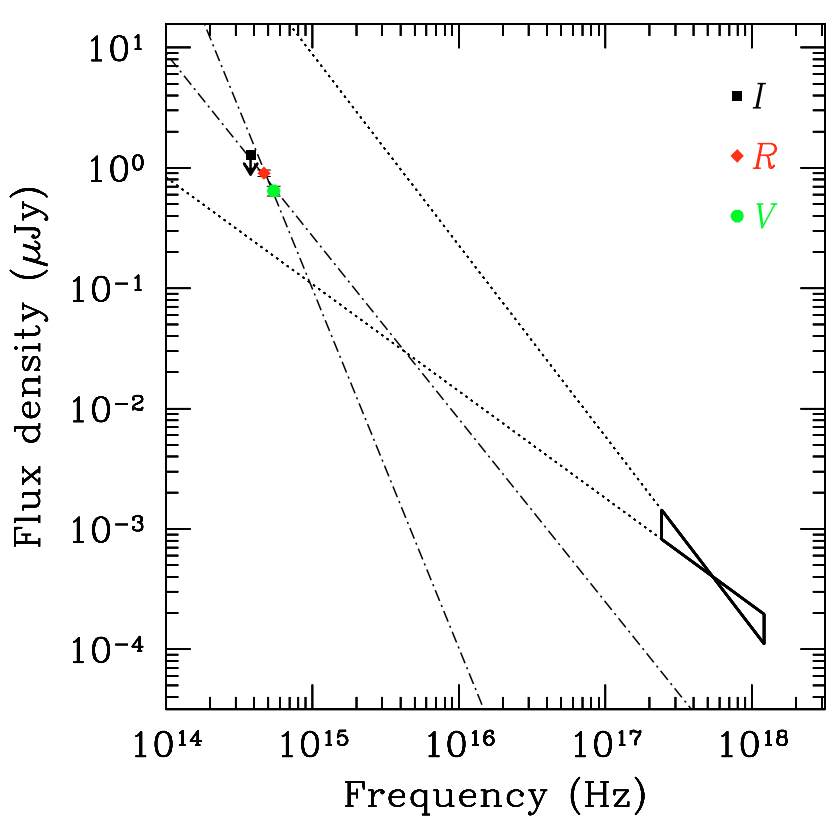

Fig. 2. Broad-band spectral energy distribution of the afterglow of GRB 050709 on Jul. 12.4 UT. The bow-tie shaped region represent the simultaneous X-ray spectrum taken from Chandra (Fox et al. 2005b). The dot-dashed and dotted lines indicate the extrapolation of the optical and X-ray spectra, respectively.

\section{Discussion}

Assuming a power-law flux decay $\left(F(t) \propto t^{-\alpha}\right)$, our observations constrain $\alpha$ to be greater than 1.0 in the $V$ band ( $3 \sigma$ upper limit). This limit is consistent with the optical decay found by Fox et al. (2005b) using HST data between 5 and $10 \mathrm{~d}$ after the GRB (see also Hjorth et al. 2005b). A similar limit was also put to the X-ray afterglow decay. Flaring activity was reported in X-ray light curve (Fox et al. 2005b). Given the limited available data, it is difficult to say whether a similar behaviour was present also in the optical band. Our measurements on Jul. 12 are nearly simultaneous with the first Chandra observation, so we can construct the broad-band spectral energy distribution (Fig. 2). The X-ray spectral index is typical for long GRB afterglows (De Pasquale et al. 2003; Nousek et al. 2005). The extrapolation of the X-ray spectrum matches the optical flux (that is, the optical-to-X-ray slope $\beta_{\mathrm{OX}}=1.1$ is consistent with the $\mathrm{X}$-ray slope $\left.\beta_{\mathrm{X}}=1.24 \pm 0.35\right)$. The spectrum corresponding to the optical colours is quite red $\left(\beta_{\text {opt }}=2.3 \pm 0.7\right)$, but given its large uncertainty it is consistent with $\beta_{\mathrm{OX}}$ at the $1.7 \sigma$ level. Small dust extinction $\left(A_{\mathrm{V}} \approx 0.2 \mathrm{mag}\right.$; Fox et al. 2005b) would make the intrinsic color bluer and fully consistent with the X-ray slope, so that the optical and X-ray emission may constitute a single component. However, the sparseness of the data prevents us from drawing any robust conclusion.

The properties of the host galaxy are intriguing. Its colours are consistent with those of an irregular galaxy at $z \approx 0.2$ (Fukugita et al. 1995), and are much bluer than those of ellipticals (like those associated with other short GRBs). Moreover, hints of morphological structure are seen in our best-seeing images. A close inspection of the spectrum shows that both the $\mathrm{H} \alpha$ and $\mathrm{H} \beta$ lines have a narrow emission component

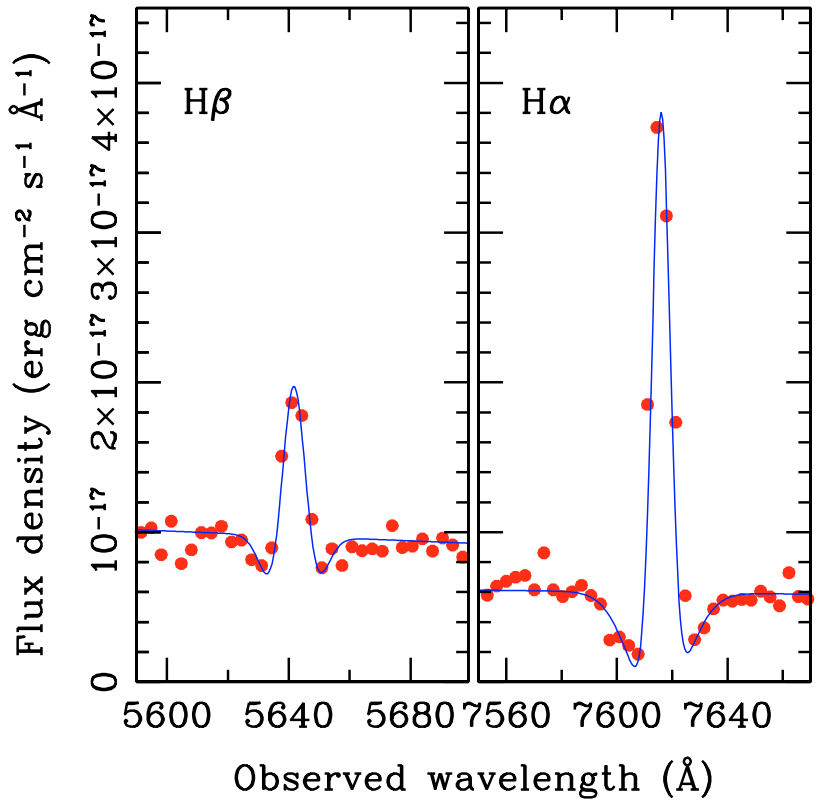

Fig. 3. Details of the GRB 050709 host galaxy spectrum close to the $\mathrm{H} \alpha$ and $\mathrm{H} \beta$ lines, showing that the narrow emission emissions are superimposed on wider absorption features.

$(F W H M<6 \AA)$ partially filling a wider $(\sim 12 \AA F W H M)$ absorption feature (Fig. 3). This classical signature (Dressler \& Gunn 1983) identifies a dominant stellar population $\sim 1$ Gyr old (mostly A-dwarf stars), together with a younger, hotter component. As indicated from the prominent nebular emission lines, star formation is still present. From the $\mathrm{H} \alpha$ and [O II] emission lines (luminosities $2.62 \times 10^{40}$ and $2.45 \times 10^{40} \mathrm{erg} \mathrm{s}^{-1}$, respectively), we infer a star formation rate of 0.21 and $0.34 M_{\odot} \mathrm{yr}^{-1}$ (Kennicutt 1998), which corresponds to $\sim 2-3.5 M_{\odot} \mathrm{yr}^{-1}$ once normalised to $L_{*}$. This is significantly less than that typically observed in long GRB host galaxies (Christensen et al. 2004), but much larger than that in the hosts of GRB $050509 \mathrm{~B}^{2}$ (Bloom et al. 2005) and GRB 050724 (Berger et al. 2005a), by factors of $>50$ and $>150$, respectively.

The most popular model for short-hard GRBs is the merger of a binary compact object system (e.g. Eichler et al. 1989). Such events can occur in an late-type, star-forming galaxy (Belczyński \& Kalogera 2001), and give rise to short GRBs (Perna \& Belczyński 2002). Since the merging timescales may be of the order of $10 \mathrm{Myr}$, small offsets between the explosion site and the galaxy core are possible. Therefore, GRB 050709 might have been produced in a tightly bound system, with a short merging time, similar to GRB 050724 (Berger et al. 2005a). However, we also note that according to the standard Faber-Jackson relation, the escape velocity from the GRB 050709 host is quite large (about $300 \mathrm{~km} \mathrm{~s}^{-1}$ ), so that only a fraction of binary systems may be able to escape its potential well. In this case, a larger delay $(\sim 1 \mathrm{Gyr})$ would be consistent both with the observed offset and with the age of the older stellar population. A large instantaneous star formation

${ }^{2}$ Note, however, that many blue galaxies were identified inside the XRT error circle. 


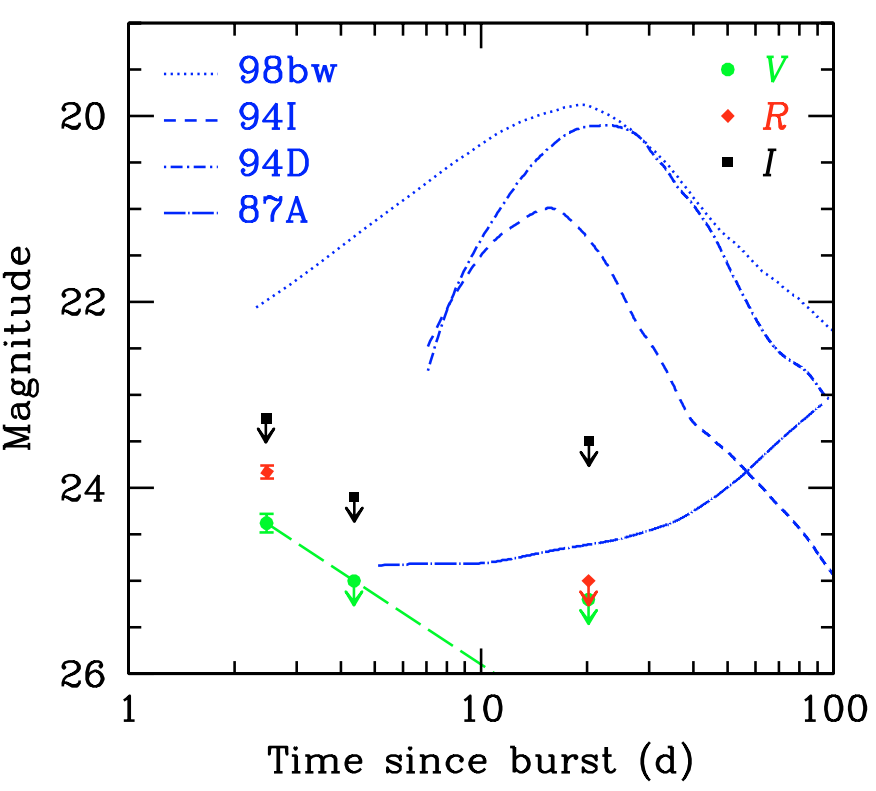

Fig. 4. Light curve of the GRB 050709 afterglow (points), compared to those of several $\mathrm{SNe}$ ( $R$ band). Zero extinction is assumed at the GRB site.

rate would not be expected in this case, even if this does not pose any problem for the merger model.

The presence of pronounced star formation activity in the host galaxy of GRB 050709, however, prompts us to investigate whether this event could be directly related to young stars. Recently, it was proposed that short GRBs may be produced by giant flares from soft gamma-ray repeaters (e.g. Hurley et al. 2005). However, the luminosity of GRB 050709 would be a factor $\sim 10^{3}$ larger than that of the giant flare from SGR 1806-20. The prompt emission properties also make this hypothesis unlikely (Villasenor et al. 2005). Furthermore, our photometry can put strong constraints on the presence of an unextinguished supernova (SN) exploded simultaneously with the GRB (see Fig. 4). Our limits impose a SN $\gtrsim 100$ times fainter than a typical type-Ia SN or a bright hypernova like SN 1998bw. Also fainter events like SN 1994I and even SN 1987A are incompatible with our data. An association with a SN seems therefore ruled out for GRB 050709 (see also Hjorth et al. 2005a,b; Fox et al. 2005b). The properties of the GRB 050709 host are however consistent with the model proposed by MacFadyen et al. (2005), which advocates a collapsing neutron star accreting from a close non-compact companion. Such model would also naturally explain the flares observed in the X-ray light curve.

Acknowledgements. D.M. thanks INAF and the Italian MIUR for support. This research was supported at OAB and OAR by ASI grant $\mathrm{I} / \mathrm{R} / 039 / 04$. We acknowledge the excellent support of the ESO staff. K.H. is grateful for support under MIT-SC-R-293291 and FDNAG5-9210. We also thank the anonymous referee for her/his valuable comments and suggestions.

\section{References}

Alard, C., \& Lupton, R. H. 1998, ApJ, 503, 325

Barthelmy, S., Chincarini, G., Burrows, D. N., et al. 2005, Nature, 438, 994

Belczyński, K., \& Kalogera, V. 2001, ApJ, 550, 183

Berger, E., Price, P. A., Cenko, S. B., et al. 2005a, Nature, 438, 988

Berger, E. 2005b, GCN 3801

Blanton, M. R., Hogg, D. W., Bahcall, N. A., et al. 2003, ApJ, 592, 819

Bloom, J. S., Prochaska, J. X., Pooley, D., et al. 2005, ApJ, in press [arXiv: astro-ph/0505480]

Castro-Tirado, A. J., de Ugarte-Postigo, A., Gorosabel, J., et al. 2005, A\&A, 439, L15

Christensen, L., Hjorth, J., \& Gorosabel, J. 2004, A\&A, 425, 913

D'Avanzo, P., Covino, S., Antonelli, L. A., et al. 2005, GCN 3690

De Pasquale, M., Piro, L., Perna, R., et al. 2003, ApJ, 592, 1018

Dressler, A., \& Gunn, J. E. 1983, ApJ, 270, 7

Eichler, D., Livio, M., Piran, T., \& Schramm, D. N. 1989, Nature, 340, 126

Fox, D. B., Frail, D. A., Cameron, P. B., et al. 2005a, GCN 3585

Fox, D. B., Frail, D. A., Price, P. A., et al. 2005b, Nature, 437, 845

Fukugita, M., Shimasaku, K., \& Ichikawa, T. 1995, PASP, 107, 945

Gehrels, N., Sarazin, C. L., O’Brien, P. T., et al. 2005, Nature, 437, 851

Gladders, M., Berger, E., Morrell, N., \& Roth, M. 2005, GCN 3798

Gorosabel, J., Castro-Tirado, A. J., Guziy, S., et al. 2005, A\&A, in press [arXiv: astro-ph/0510141]

Hjorth, J., Sollerman, J., Gorosabel, J., et al. 2005a, ApJ, 630, L117

Hjorth, J., Watson, D., Fynbo, J. P. U., et al. 2005b, Nature, 437, 859

Hurley, K., Berger, E., Castro-Tirado, A. J., et al. 2002, ApJ, 567, 447

Hurley, K., Boggs, S. E., Smith, D. M., et al. 2005, Nature, 434, 1098

Jensen, B. L., Jörgensen, U. G., Hjorth, J., et al. 2005, GCN 3589

Kennicutt, R. C. Jr 1998, ARA\&A, 36, 189

King, A., O’Brien, P. T., Goad, M. R., et al. 2005, ApJ, 630, L113

Kouveliotou, C., Meegan, C. A., Fishman, G. J., et al. 1993, ApJ, 413, 101

MacFadyen, A. I., Ramirez-Ruiz, E., \& Zhang, W. 2005 [arXiv: astro-ph/0510192]

Perna, R., \& Belcźynski, K. 2002, ApJ, 570, 252

Perna, R., Armitage, P. J., \& Zhang, B. 2005, ApJ, 636, L29

Nousek, J. A., Kouveliotou, C., Grupe, D., et al. 2005, ApJ, submitted [arXiv: astro-ph/0508332]

Price, P. A., Roth, K., \& Fox, D. W. 2005a, GCN 3605

Price, P. A., Jensen, B. L., Jörgensen, U. G., et al. 2005b, GCN 3612

Prochaska, J. X., Bloom, J. S., Chen, H.-W., et al. 2005, ApJ, submitted [arXiv: astro-ph/0510022]

Retter, A., Barbier, L., Barthelmy, S., et al. 2005, GCN 3788

Villasenor, J., Lamb, D. Q., Ricker, G. R., et al. 2005, Nature, 437, 855 